\title{
Erratum to: Escape from the Island: Grammaticality and (Reduced) Acceptability of $w h$-island Violations in Danish
}

\author{
Ken Ramshøj Christensen • Johannes Kizach • \\ Anne Mette Nyvad
}

Published online: 6 July 2012

(C) Springer Science+Business Media, LLC 2012

\section{Erratum to: J Psycholinguist Res DOI 10.1007/s10936-012-9210-x}

The analyses in the original paper were not repeated measures ANOVAs. The repeated measures analysis, however, does not change the overall results and therefore has no effect on the chain of argumentation and no effect on the conclusions. The aim of this erratum is only to put the record straight and to present the proper analysis.

In experiment 1 , a repeated measures ANOVA $(F 1(8,472)=309.20, p<.001$; $F 2(8,120)=1130.37, p<.001)$ and post hoc Bonferroni-corrected $t$-tests confirmed that conditions could be ranked with respect to acceptability as follows: $\{$ BASE(VS/SV), SHORT (ARG/ADJ) $\}>$ LONG (ARG) $>$ LONG (ADJ) $>$ ACROSS (ARG/ADJ) $>*$ Doubly-filled Spec-CP. As before, there were no significant differences within or between the BASE conditions and the SHORT conditions $(p>.27)$.

In experiment 2 , repeated measures $(F 1(4,116)=270.88, p<.001 ; F 2(4,44)=36.53$, $p<.001)$ and post hoc Bonferroni-corrected $t$-tests confirmed the acceptability hierarchy found in the original analysis: LONG (ARG $>$ ADJ) $>$ ACROSS (ARG/ADJ) $>$ ANOMALY $(p<.001)$. There was no significant ARG/ADJ asymmetry within the Across condition $(p=.07)$. In terms of response time, two contrasts revealed a significant difference, namely ACROSS (ADJ) $>$ LONG (ADJ) $(p=.03)$ and LONG (ADJ) $>$ ANOMALY $(p=.03)$; in the original paper, the latter contrast was only marginal. All other contrasts revealed no significant differences $(p=1.00)$.

In experiment 1, 17 males and 43 females participated (not 16 and 44).

The online version of the original article can be found under doi:10.1007/s10936-012-9210-x.

K. R. Christensen

Center for Functionally Integrative Neuroscience, Aarhus University Hospital,

Aarhus C, Denmark

K. R. Christensen $(\bowtie) \cdot$ J. Kizach · A. M. Nyvad

Department of Aesthetics and Communication, Aarhus University,

Jens Chr. Skous Vej 2, 8000 Aarhus C, Denmark

e-mail: Ken@cfin.dk 